\title{
Revealing the cosmic reionization history with fast radio bursts in the era of Square Kilometre Array
}

\author{
Tetsuya Hashimoto ${ }^{\circledR}, 1,2 \star$ Tomotsugu Goto, ${ }^{1}$ Ting-Yi Lu ${ }^{\circledR},{ }^{1}$ Alvina Y. L. On ${ }^{\circledR}$, ${ }^{1,2,3}$ Daryl Joe \\ D. Santos ${ }^{\circledR},{ }^{1}$ Seong Jin Kim ${ }^{\circledR},{ }^{1}$ Ece Kilerci Eser ${ }^{\circledR},{ }^{4}$ Simon C.-C. Ho ${ }^{\circledR}$, ${ }^{1}$ Tiger Y.-Y. Hsiao ${ }^{1}$ and Leo \\ Y.-W. Lin ${ }^{5}$ \\ ${ }^{1}$ Institute of Astronomy, National Tsing Hua University, 101, Section 2. Kuang-Fu Road, Hsinchu 30013, Taiwan \\ ${ }^{2}$ Centre for Informatics and Computation in Astronomy (CICA), National Tsing Hua University, 101, Section 2. Kuang-Fu Road, Hsinchu 30013, Taiwan \\ ${ }^{3}$ Mullard Space Science Laboratory, University College London, Holmbury St Mary, Surrey RH5 6NT, UK \\ ${ }^{4}$ Faculty of Engineering and Natural Sciences, Sabancı University, 34956 Istanbul, Turkey \\ ${ }^{5}$ Department of Physics, National Tsing Hua University, 101, Section 2. Kuang-Fu Road, Hsinchu 30013, Taiwan
}

Accepted 2021 January 18. Received 2020 December 24; in original form 2020 October 22

\begin{abstract}
Revealing the cosmic reionization history is at the frontier of extragalactic astronomy. The power spectrum of the cosmic microwave background $(\mathrm{CMB})$ polarization can be used to constrain the reionization history. Here, we propose a CMBindependent method using fast radio bursts (FRBs) to directly measure the ionization fraction of the intergalactic medium (IGM) as a function of redshift. FRBs are new astronomical transients with millisecond time-scales. Their dispersion measure $\left(\mathrm{DM}_{\mathrm{IGM}}\right)$ is an indicator of the amount of ionized material in the IGM. Since the differential of $\mathrm{DM}_{\mathrm{IGM}}$ against redshift is proportional to the ionization fraction, our method allows us to directly measure the reionization history without any assumption on its functional shape. As a proof of concept, we constructed mock non-repeating FRB sources to be detected with the Square Kilometre Array, assuming three different reionization histories with the same optical depth of Thomson scattering. We considered three cases of redshift measurements: (A) spectroscopic redshift for all mock data, (B) spectroscopic redshift for 10 per cent of mock data, and (C) redshift estimated from an empirical relation of FRBs between their time-integrated luminosity and rest-frame intrinsic duration. In all cases, the reionization histories are consistently reconstructed from the mock FRB data using our method. Our results demonstrate the capability of future FRBs in constraining the reionization history.
\end{abstract}

Key words: stars: luminosity function, mass function-stars: magnetars - stars: neutron-dark ages, reionization, first starsearly Universe - radio continuum: transients.

\section{INTRODUCTION}

The cosmic (hydrogen) reionization is the latest phase transition of the Universe from neutral to ionized state (e.g. Barkana \& Loeb 2001). During the reionization epoch, the intergalactic medium (IGM) was presumably reionized by strong radiation from the first stars and the first galaxies (e.g. Bouwens et al. 2012; Finkelstein et al. 2012; Duncan \& Conselice 2015; Robertson et al. 2015; Naidu et al. 2020 ) with probably minor contributions from quasars (QSOs, e.g. Onoue et al. 2017; Yoshiura et al. 2017; Hassan et al. 2018; Matsuoka et al. 2018). Revealing how and when the reionization happened is at the forefront of extragalactic astronomy.

Several methods have been proposed to investigate the cosmic reionization. Using Ly $\alpha$ absorption and its damping wing is one of the traditional methods. High-redshift (high- $z$ ) bright sources such as QSOs and optical afterglows of long gamma-ray bursts (LGRBs) are used as background light sources (e.g. Fan et al. 2002; Goto 2006; Becker et al. 2015; Barnett et al. 2017; Bosman et al. 2018; Lu et al. 2020; Totani et al. 2014; Hartoog et al. 2015; Melandri et al.

${ }^{\star}$ E-mail: tetsuya@phys.nthu.edu.tw
2015). Their light is absorbed by the intervening IGM between the source and the observer, producing Ly $\alpha$ absorption features which are imprinted in their spectra. This allows us to measure the ionization fraction in the IGM as a function of redshift. This method does not assume any model of the reionization in calculating the ionization fraction from the observed Ly $\alpha$ absorption. The tail end of cosmic reionization is largely confirmed to be $z \sim 6$ by high-redshift QSOs and LGRB afterglows in previous studies (e.g. Fan et al. 2002, 2006; Totani et al. 2006). However, applying this method beyond $z \sim 7$ is challenging because the number densities of bright highz QSOs and LGRBs rapidly decline towards higher redshifts (e.g. Matsuoka et al. 2018; Wang et al. 2019; Perley et al. 2016). In addition, the observed spectra require both high spectral resolution and high signal-to-noise ratio, given that the transmitted light is very faint due to the strong $\operatorname{Ly} \alpha$ absorption. This observational difficulty hampers accurate measurements of ionization fraction at $z \gtrsim 7$, placing relatively large uncertainties (e.g. Bañados et al. 2018; Yang et al. 2020).

On the other hand, cosmic microwave background (CMB) observations can reach high redshifts up to the recombination epoch of the Universe (i.e. $z \sim 1100$ ) beyond QSOs and LGRBs (e.g. 
Hinshaw et al. 2013; Planck Collaboration VI 2020). CMB allows us to measure the optical depth of Thomson scattering of free electrons in the IGM $\left(\tau_{\mathrm{CMB}}\right)$. Since $\tau_{\mathrm{CMB}}$ is an integration over redshift from $z$ $=0$ to $\sim 1100$, it also depends on the epoch of reionization. Recently, the optical depth was estimated to be $\tau_{\mathrm{CMB}}=0.059 \pm 0.006$ from Planck observations (Pagano et al. 2020). This value corresponds to an averaged reionization redshift at $z=8.14 \pm 0.61$, assuming a Tanh reionization history (Pagano et al. 2020). This estimate is largely consistent with the end of reionization epoch at $z \sim 6$ as revealed by QSOs and LGRBs (e.g. Fan et al. 2002, 2006; Totani et al. 2006). However, solely using $\tau_{\mathrm{CMB}}$ is not sensitive to the detailed ionization history, that is, the ionization fraction as a function of redshift, because it measures only the amount of electron scattering integrated over redshift. The shape of the power spectrum of CMB polarization encodes information on the detailed redshift evolution of the ionization fraction (e.g. Zaldarriaga, Spergel \& Seljak 1997; Kaplinghat et al. 2003; Planck Collaboration VI 2020). Therefore, the CMB polarization in principle can constrain the early reionization at $z \sim 15-30$. The optical depth between $z=15$ and 30 is constrained to $\tau_{\mathrm{CMB}}(15,30)<0.007$ (Planck Collaboration VI 2020).

21-cm absorption or emission features of atomic hydrogen in an all-sky radio spectrum is also a powerful tracer of cosmic reionization (e.g. Furlanetto, Oh \& Briggs 2006; Pritchard \& Loeb 2012; Barkana 2016). The absorption feature appears when the 21-cm spin temperature is lower than the CMB temperature in the early Universe. This condition is satisfied when gas temperature is coupled with the $21-\mathrm{cm}$ spin temperature due to resonant scattering of Ly $\alpha$ photons emitted from the first stars and galaxies (e.g. Bowman et al. 2018). Over time, the gas and coupled $21-\mathrm{cm}$ spin temperatures are expected to become higher than the $\mathrm{CMB}$ temperature due to the heating effects by galaxies and active galactic nuclei, ending the absorption feature. Therefore, the $21-\mathrm{cm}$ absorption is sensitive to the beginning of cosmic reionization before the majority of the IGM starts to become reionized. Bowman et al. (2018) claimed the detection of the 21-cm absorption around $z \sim 17$ from observations with the Experiment to Detect the Global Epoch of Reionization Signature (EDGES) low-band instrument (Bowman, Rogers \& Hewitt 2008; Rogers \& Bowman 2008). The absorption feature spans from $z \sim$ 15 to $\sim 20$. The $z \sim 20$ and $\sim 15$ edges of the absorption feature correspond to the redshift at which the $21-\mathrm{cm}$ spin temperature became lower than the $\mathrm{CMB}$ temperature and the redshift at which the gas temperature exceeded the CMB temperature, respectively. This implies that cosmic reionization has most likely started at $z \lesssim 20$. However, we caution that the EDGES observation has large measurement errors. Therefore, the actual redshifts at which the signal crosses zero might be very different from the abrupt drops. In addition, the EDGES signal might be non-cosmological, in which case there is no associated redshift. During reionization, the 21-cm spin temperature becomes much higher than the CMB temperature, which causes the 21-cm emission feature. The 21$\mathrm{cm}$ emission directly traces the ionization fraction in the IGM (e.g. Bowman \& Rogers 2010). However, no clear detection of the $21-\mathrm{cm}$ emission in the IGM at the reionization epoch has been reported yet.

None of these methods has provided a complete picture of the cosmic reionization history yet. In contrast, fast radio bursts (FRBs) have a potential to allow us to overcome the difficulties mentioned above. FRB is a new type of astronomical transients, which is a bright burst in radio (order of Jy) with a millisecond time-scale (e.g. Lorimer et al. 2007). A unique observable of FRBs is the dispersion measure (DM), which is characterized by a time lag of burst arrival depending on observed frequencies. The DM is an indicator of the amount of ionized material between the source of FRB and the observer. The majority of FRBs shows an observed DM $\left(\mathrm{DM}_{\mathrm{obs}}\right) \gtrsim 300 \mathrm{pc} \mathrm{cm}^{-3}$, indicating that the sources must be extragalactic (e.g. Cordes \& Chatterjee 2019; Hashimoto et al. 2020a). Since the DM is sensitive to the ionized IGM, some studies (e.g. Caleb, Flynn \& Stappers 2019; Kit Lau et al. 2020; Linder 2020) proposed to use FRBs to constrain the 'helium reionization' at $z \sim 3-4$ (e.g. Becker et al. 2011) with future FRBs. However, the sample size and redshift range of current FRB data are too small to constrain the reionization history (e.g. Petroff et al. 2016). The Square Kilometre Array (SKA) is expected to detect hundreds to thousands of FRBs at $z \gtrsim 6$ (e.g. Fialkov \& Loeb 2017; Hashimoto et al. 2020b), which motivates studies on the hydrogen reionization at $z \gtrsim 6$ using future DM measurements (e.g. Ioka 2003; Inoue 2004; Fialkov \& Loeb 2016; Dai \& Xia 2020).

FRBs provide a new method to measure the amount of ionized IGM independent of the CMB. The CMB has only one source redshift and thus can only probe the ionization fraction along the line of sight all the way out to $z \sim 1100$. However, if high- $z$. FRBs exist, they are sources at multiple redshifts and can probe the ionized fraction out to different redshifts (e.g. Ioka 2003; Inoue 2004; Fialkov \& Loeb 2016).

Zheng et al. (2014) pointed out that the differential of averaged $\mathrm{DM}_{\mathrm{IGM}}$ against redshift (i.e. $\mathrm{d}\left\langle\mathrm{DM}_{\mathrm{IGM}}\right\rangle / \mathrm{d} z$ ) is more sensitive to the reionization history than the $\mathrm{DM}_{\mathrm{IGM}}$ itself. Motivated by the previous work, we propose to use $d\left\langle\mathrm{DM}_{\mathrm{IGM}}\right\rangle / \mathrm{d} z$, which will be available for future FRBs. The $d\left\langle\mathrm{DM}_{\mathrm{IGM}}\right\rangle / \mathrm{d} z$ allows us to directly measure the ionization fraction without any assumption on a functional shape of the reionization history.

The structure of this paper is as follows. In Section 2, we describe the ionization fraction as a function of redshift derived from $\mathrm{d}\left\langle\mathrm{DM}_{\mathrm{IGM}}\right\rangle / \mathrm{d} z$. We generate mock FRB data to be detected with the SKA in Section 3. In Section 4, we show ionization fractions as a function of redshift reconstructed from the mock FRB data, followed by discussions in Section 5 and conclusions in Section 6 . Throughout this paper, we assume the Planck15 cosmology (Planck Collaboration XIII 2016) as a fiducial model, that is, $\Lambda$-cold dark matter cosmology with $\left(\Omega_{m}, \Omega_{\Lambda}, \Omega_{b}, h\right)=(0.307,0.693,0.0486$, $0.677)$, unless otherwise mentioned.

\section{DIFFERENTIAL OF DISPERSION MEASURE}

The $\tau_{\mathrm{CMB}}$ and $\mathrm{DM}_{\mathrm{IGM}}$ can be measured from the CMB and FRBs, respectively. Both quantities integrate the electron density $\left(n_{\mathrm{e}}\right)$ in the IGM as follows.

$\tau_{\mathrm{CMB}}=\int_{z=0}^{1100} \sigma_{T} n_{\mathrm{e}}(z) \mathrm{d} l$

and

$\operatorname{DM}_{\mathrm{IGM}}(z)=\int_{0}^{z} \frac{n_{\mathrm{e}}\left(z^{\prime}\right)}{1+z^{\prime}} \mathrm{d} l^{\prime}$,

where $\sigma_{T}=6.65 \times 10^{-25} \mathrm{~cm}^{2}$ is the Thomson scattering crosssection, $\mathrm{d} l=c \mathrm{~d} t=\frac{-c \mathrm{~d} z}{(1+z) H(z)}$ and $d l^{\prime}=\frac{-c d z^{\prime}}{\left(1+z^{\prime}\right) H\left(z^{\prime}\right)}$ are the differentials of the proper distances, and $H(z)$ is the Hubble parameter. Since the CMB observations measure the integration of $n_{\mathrm{e}}$ from the recombination epoch $(z \sim 1100)$ to $z=0$, solely using $\tau_{\mathrm{CMB}}$ is sensitive to only the averaged epoch of reionization. In contrast to $\tau_{\mathrm{CMB}}$, FRBs allow us to measure the integration of $n_{\mathrm{e}}$ at different redshifts by identifying their host galaxies (see also Section 3.3). At a fixed redshift, the $\mathrm{DM}_{\mathrm{IGM}}$ will vary over different lines of sight due to the density fluctuation of IGM. For a flat universe, the $\mathrm{DM}_{\mathrm{IGM}}$ averaged over the different lines of sight is expressed as follows (e.g. 
Zhou et al. 2014).

$$
\begin{aligned}
& \left\langle\mathrm{DM}_{\mathrm{IGM}}\right\rangle(z)=\Omega_{\mathrm{b}} \frac{3 H_{0} c}{8 \pi G m_{\mathrm{p}}} \times \\
& \int_{0}^{z} \frac{\left(1+z^{\prime}\right) f_{\mathrm{IGM}}\left(z^{\prime}\right) M_{\mathrm{e}}\left(z^{\prime}\right)}{\left\{\Omega_{\mathrm{m}}\left(1+z^{\prime}\right)^{3}+\Omega_{\Lambda}\left(1+z^{\prime}\right)^{3\left[1+w\left(z^{\prime}\right)\right]}\right\}^{1 / 2}} \mathrm{~d} z^{\prime}
\end{aligned}
$$

and

$$
\begin{aligned}
M_{\mathrm{e}}\left(z^{\prime}\right)= & Y_{\mathrm{H}} X_{\mathrm{eH} \mathrm{II}}\left(z^{\prime}\right)+\frac{1}{4} Y_{\mathrm{p}} X_{\mathrm{eHe} \text { II }}\left(z^{\prime}\right) \\
& +\frac{1}{2} Y_{\mathrm{p}} X_{\mathrm{eHe} \text { III }}\left(z^{\prime}\right),
\end{aligned}
$$

where $X_{\mathrm{eHII}}, X_{\mathrm{eHe} I I}$, and $X_{\mathrm{eHe} \mathrm{III}}$ are the ionization fractions of intergalactic H II, He II, and He III, respectively. These are defined as $n_{\mathrm{H} \mathrm{II}} / n_{\mathrm{H}}, n_{\mathrm{He} \mathrm{II}} / n_{\mathrm{He}}$, and $n_{\mathrm{He} \text { III }} / n_{\mathrm{He}}$, respectively, where $n_{\mathrm{X}}$ represents the number density of element $\mathrm{X}$ in the IGM. The neutral, singly and doubly ionized elements are denoted by subscripts I, II, and III, respectively. $Y_{\mathrm{H}}=\frac{3}{4}$ and $Y_{\mathrm{p}}=\frac{1}{4}$ are the mass fractions of $\mathrm{H}$ and $\mathrm{He}$, respectively. $f_{\mathrm{IGM}}$ is the mass fraction of baryons in the IGM. $w(z)$ corresponds to the equation of state of dark energy. We assume a constant dark energy, that is, $w=-1$ (Chevallier \& Polarski 2001; Linder 2003). Following Zhou et al. (2014), we adopt $f_{\mathrm{IGM}}=0.9$ at $z>1.5$ and $f_{\mathrm{IGM}}=0.053 z+0.82$ at $z \leq 1.5$ (e.g. Meiksin 2009; Shull, Smith \& Danforth 2012). The parameter $f_{\mathrm{IGM}}$ is necessary to take into account the amount of intergalactic ionized material traced by the FRBs.

In this work, we propose to differentiate the $\left\langle\mathrm{DM}_{\mathrm{IGM}}\right\rangle$ against redshift, that is, $\frac{\mathrm{d}\left\langle\mathrm{DM}_{\mathrm{IGM}}\right\rangle(z)}{\mathrm{d} z}$, to measure $X_{\mathrm{eHII}}$. At the hydrogen reionization epoch, $X_{\text {eHe II }}$ can be approximated as $X_{\text {eH II }}$ (e.g. Dai \& Xia 2020) because both neutral hydrogen and neutral helium are singly ionized by ionizing photons radiated from star-forming galaxies. Supposing that the helium reionization happened at $z \sim$ 3-4 (e.g. Becker et al. 2011), $X_{\text {eHe III }} \sim 0$ at $z>4$. Therefore, by differentiating equation (3) against redshift, $X_{\mathrm{eH} \text { II }}$ at the hydrogen reionization epoch is expressed as

$$
\begin{aligned}
X_{\mathrm{eH} \mathrm{II}}(z)= & \frac{\mathrm{d}\left\langle\mathrm{DM}_{\mathrm{IGM}}\right\rangle(z)}{\mathrm{d} z} \frac{3 H_{0} c \Omega_{b}}{8 \pi G m_{p}} \frac{1}{Y_{\mathrm{H}}+\frac{1}{4} Y_{\mathrm{p}}} \\
& \times \frac{\left\{\Omega_{\mathrm{m}}(1+z)^{3}+\Omega_{\Lambda}(1+z)^{3[1+w(z)]}\right\}^{1 / 2}}{(1+z) f_{\mathrm{IGM}}(z)} .
\end{aligned}
$$

Since $\frac{\mathrm{d}\left\langle\mathrm{DM}_{\mathrm{IGM}}\right\rangle(z)}{\mathrm{d} z}$ will be measured by a slope in the $\mathrm{DM}_{\mathrm{IGM}}-z$ parameter space, equation (5) allows us to directly measure $X_{\mathrm{eH} \mathrm{II}}(z)$ without any assumption on a functional shape of the reionization history. In the following sections, we demonstrate how this method can work in the SKA era using mock FRB data.

\section{MOCK FRB DATA}

\subsection{Reionization histories}

To test the method, we consider three different cosmic reionization histories with the same $\tau_{\mathrm{CMB}}$ as prior assumptions. We describe their parametrizations in the following sections. The parameters in the three reionization histories are selected such that $\tau_{\mathrm{CMB}}=0.05$, which is calculated using equation (1). This value is consistent with one of the recent $\mathrm{CMB}$ constraints, $\tau_{\mathrm{CMB}}=0.054 \pm 0.007$, within the uncertainty (Planck Collaboration VI 2020). Therefore, solely using $\tau_{\mathrm{CMB}}$ is not sensitive to distinguishing between the three reionization histories presented in this work.

\subsubsection{Tanh reionization history}

The first case is a Tanh reionization. This model is conventionally used when the reionization epoch is constrained by $\tau_{\mathrm{CMB}}$ (e.g. Lewis 2008). In this model, the ionization fraction of intergalactic hydrogen, $X_{\mathrm{e}, \mathrm{HII}}$, is parametrized as follows.

$X_{\mathrm{eH} \mathrm{II}}^{\mathrm{tanh}}(z)=1-g\left(z, z_{\mathrm{re}}, \Delta z_{\mathrm{tanh}}\right)$,

where

$g\left(z, z_{\mathrm{re}}, \Delta z_{\mathrm{tanh}}\right)=\frac{1}{2}\left[1+\tanh \left(\frac{(1+z)^{3 / 2}-\left(1+z_{\mathrm{re}}\right)^{3 / 2}}{1.5 \sqrt{1+z_{\mathrm{re}}} \Delta z_{\mathrm{tanh}}}\right)\right]$.

The parameters, $z_{\text {re }}$ and $\Delta z_{\text {tanh }}$, represent the redshift at which $X_{\mathrm{eH} \text { II }}^{\mathrm{tanh}}(z)$ becomes 0.5 (i.e. $\left.X_{\mathrm{eH} \mathrm{II}}^{\mathrm{tanh}}\left(z_{\mathrm{re}}\right)=0.5\right)$ and the redshift width of the phase transition from neutral to ionized states, respectively. We adopt $z_{\text {re }}=7.8$ and $\Delta z_{\text {tanh }}=0.5$. The model is shown in Fig. 1(a) in the blue line.

Since both neutral hydrogen and neutral helium are singly ionized by ionizing photons from star-forming galaxies, we assume the same functions as the hydrogen reionization for neutral helium until the $\mathrm{He}$ II is ionized into He III at the helium reionization epoch. Therefore, the abundance of $\mathrm{He}$ II decreases towards lower redshifts at the helium reionization epoch, while He III increases. The ionization fractions of He II are parametrized as follows:

$X_{\mathrm{eHe} \text { II }}^{\mathrm{tanh}}(z)=-g\left(z, z_{\mathrm{re}}, \Delta z_{\mathrm{tanh}}\right)+g\left(z, z_{\mathrm{re}, \mathrm{He}}, \Delta z_{\mathrm{He}}\right)$,

where $z_{\mathrm{re}, \mathrm{He}}$ and $\Delta z_{\mathrm{He}}$ represent the epoch of helium reionization and its redshift width, respectively. We adopt $z_{\mathrm{re}, \mathrm{He}}=3.0$ and $\Delta z_{\mathrm{He}}$ $=0.05$. The model is shown in Fig. 1(b) in the blue line.

\subsubsection{Asymmetric reionization history}

The second case is a redshift-asymmetric model. Numerically simulated reionization histories could be parametrized better by the redshift-asymmetric function (Douspis et al. 2015). This model is parametrized as follows.

$X_{\mathrm{eHII}}^{\text {asym. }}(z)= \begin{cases}1-\frac{\left(1-X_{\mathrm{e}, 6}\right)(1+z)^{3}}{(1+6)^{3}} & (z \leq 6) \\ X_{\mathrm{e}, 6} \mathrm{e}^{\alpha(6-z)} & (z>6),\end{cases}$

where $X_{\mathrm{e}, 6}$ is ionization fraction at $z=6$. The exponent $\alpha$ is a parameter to describe the duration of transition from neutral to ionized phases. We adopt $X_{\mathrm{e}, \mathrm{H}}=0.9$ and $\alpha=0.5$. The model is shown in Fig. 1(a) in the green line.

In a similar way to the Tanh reionization, the ionization fraction of $\mathrm{He}$ II is approximated as the hydrogen ionization fraction as follows:

[

$X_{\mathrm{eHe} \text { II }}^{\mathrm{asym} .}(z)=\left\{\begin{array}{lc}g\left(z, z_{\mathrm{re}, \mathrm{He}}, \Delta z_{\mathrm{He}}\right) & (z<3) \\ -\frac{\left(1-X_{\mathrm{e}, 6)(1+z)^{3}}\right.}{(1+6)^{3}}+g\left(z, z_{\mathrm{re}, \mathrm{He}}, \Delta z_{\mathrm{He}}\right) & (3 \leq z \leq 6) \\ X_{\mathrm{e}, 6} \mathrm{e}^{\alpha(6-z)}-\left\{1-g\left(z, z_{\mathrm{re}, \mathrm{He}}, \Delta z_{\mathrm{He}}\right)\right\} & (z>6) .\end{array}\right.$

The model is shown in Fig. 1(b) in the green line.

\subsubsection{Double reionization history}

The third case is a double reionization history. According to reionization models, the double reionization processes may have happened at $z \sim 10-15$ and $\sim 6$ due to Population III stars and normal star-forming galaxies, respectively (e.g. Cen 2003; Salvador-Solé et al. 2017). The double reionization at $z>15$ is highly disfavoured by the CMB data (e.g. Planck Collaboration VI 2020). While the double reionization 

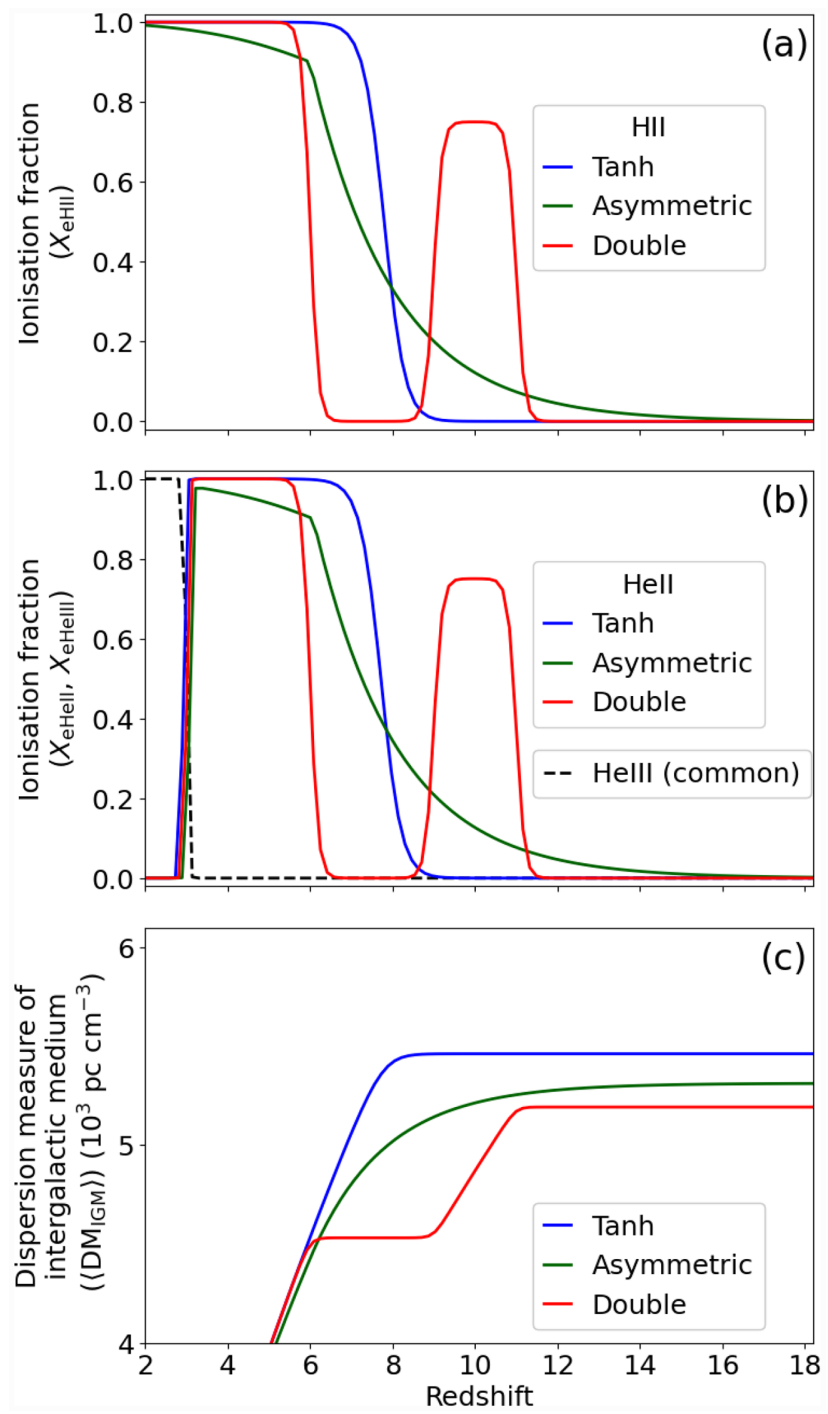

Figure 1. (a) Assumed cosmic reionization histories with the same optical depth of Thomson scattering, $\tau_{\mathrm{CMB}}=0.05$. Their ionization fractions of hydrogen are shown in different colours. (b) Same as top except for ionization fractions of $\mathrm{He}$ II and He III. The blue and green lines are slightly shifted along the horizontal axis for a visual purpose. The same ionization fraction of He III is commonly assumed for the three reionization histories. (c) The DM of the IGM as a function of redshift. The DM is averaged over the density fluctuation of the IGM at each redshift $\left(\left\langle\mathrm{DM}_{\mathrm{IGM}}\right\rangle\right)$. The coloured solid lines correspond to the three ionization histories shown in the panels (a) and (b).

is not the main-stream model, it is still useful to demonstrate the feasibility of future FRBs which can reveal detailed substructures of the cosmic reionization history if such substructures exist. In this work, we parametrize the double reionization history using Tanh functions (i.e. equation 7) as follows:

$$
\begin{aligned}
X_{\mathrm{eH} I \mathrm{II}}^{\text {double }}(z)=1 & +A\left\{-g\left(z, z_{\text {re } 1, \Delta \mathrm{z}_{\text {double }}}\right)+g\left(z, z_{\mathrm{re} 2, \Delta \mathrm{z}_{\text {double }}}\right)\right\} \\
& -g\left(z, z_{\mathrm{re} 3, \Delta \mathrm{z}_{\text {double }}}\right) .
\end{aligned}
$$

The redshift parameters, $z_{\mathrm{re} 1}, z_{\mathrm{re} 2}$, and $z_{\mathrm{re} 3}$, correspond to the beginning and the end of the first reionization, and the beginning of the second reionization, respectively. The redshift width of the start and end of reionization is described by $\Delta z_{\text {double }}$. The maximum ionization fraction during the first reionization is parametrized by $A$.
We adopt $z_{\text {re1 }}=11.0, z_{\text {re2 }}=9.0, z_{\text {re3 }}=6.0, \Delta z_{\text {double }}=0.2$, and $A=$ 0.75. The model is shown in Fig. 1(a) in the red line.

In a similar way to the Tanh reionization, the ionization fraction of He II is approximated as the hydrogen ionization fraction as follows:

$$
\begin{aligned}
X_{\text {eHe II }}^{\text {double }}(z)= & 1+A\left\{-g\left(z, z_{\text {re } 1}, \Delta z_{\text {double }}\right)+g\left(z, z_{\text {re } 2}, \Delta z_{\text {double }}\right)\right\} \\
& -g\left(z, z_{\text {re3 } 3}, \Delta z_{\text {double }}\right)+g\left(z, z_{\text {re }, \mathrm{He}}, \Delta z_{\text {He }}\right) .
\end{aligned}
$$

The model is shown in Fig. 1(b) in the red line.

\subsubsection{Helium reionization}

In this work, a Tanh helium reionization history is commonly assumed among the three cases of hydrogen reionization histories. This is because the helium reionization has a negligible effect on $\mathrm{DM}_{\mathrm{IGM}}$ at the hydrogen reionization epoch which we focus on in this work. The ionization fraction of $\mathrm{He}$ III is commonly parametrized as follows:

$X_{\text {eHe III }}^{\text {common }}(z)=1-g\left(z, z_{\mathrm{re}, \mathrm{He}}, \Delta z_{\mathrm{He}}\right)$.

This function is shown by the black dashed line in Fig. 1(b).

The $\left\langle\mathrm{DM}_{\mathrm{IGM}}\right\rangle$ of three reionization histories are shown in Fig. 1(c), which are calculated using equation (3).

\subsection{Number of FRBs to be detected with the SKA}

Recently, the number of FRBs to be detected with the SKA Mid frequency aperture array phase 2 (e.g. Torchinsky et al. 2016) was predicted by Hashimoto et al. (2020b) with a $10 \sigma$ detection threshold. While their work was limited up to $z=14$ (Hashimoto et al. 2020b), the first galaxies may appear as early as $z \sim 20$ (e.g. Springel et al. 2005; Lacey et al. 2011; Bromm \& Yoshida 2011). Therefore, in this work, we extended their calculations towards higher redshifts up to $z=20$ under the same assumptions as follows. We consider non-repeating FRBs and their luminosity functions (LFs) which are proportional to either the cosmic stellarmass density (CSMD; López Fernández et al. 2018) or cosmic star formation-rate density (CSFRD; Madau \& Fragos 2017, see also Hashimoto et al. 2020c, for the redshift evolution of empirically derived FRB LFs). We use non-repeating FRBs because they are thought to dominate the future FRB sample due to their high number density and brightness (Hashimoto et al. 2020b). A characteristic spectral index of FRBs is assumed to be $\alpha=-0.3$, which was constrained by broad-band observations between the Green Bank Telescope North Celestial Cap survey at $0.3-0.4$ GHz and Parkes surveys at $1.4 \mathrm{GHz}$ (Chawla et al. 2017). The pulse broadening by scattering is empirically included in the calculations (see Hashimoto et al. 2020b, for details). No free-free absorption is assumed in the vicinity of FRB progenitors in this work. The adopted Galactic coordinates of FRBs are $(\ell, b)=\left(45.0^{\circ},-90.0^{\circ}\right)$. Note that a small difference in Galactic coordinates negligibly affect the FRB detection (Hashimoto et al. 2020b). The observed frequency of SKA is centred at $0.65 \mathrm{GHz}$ (e.g. Torchinsky et al. 2016). The predicted number of FRBs with the SKA is shown in Fig. 2 as a function of redshift. We note that the $10 \sigma$ detection threshold is utilized for the predictions. This threshold is conventionally used for currently detected FRBs. Therefore, in this work, we consider high-z FRBs with such high detection significance only.

\subsection{Generating mock data}

We generate mock data which correspond to non-repeating FRBs to be detected with SKA's one-year observations. At each redshift bin 


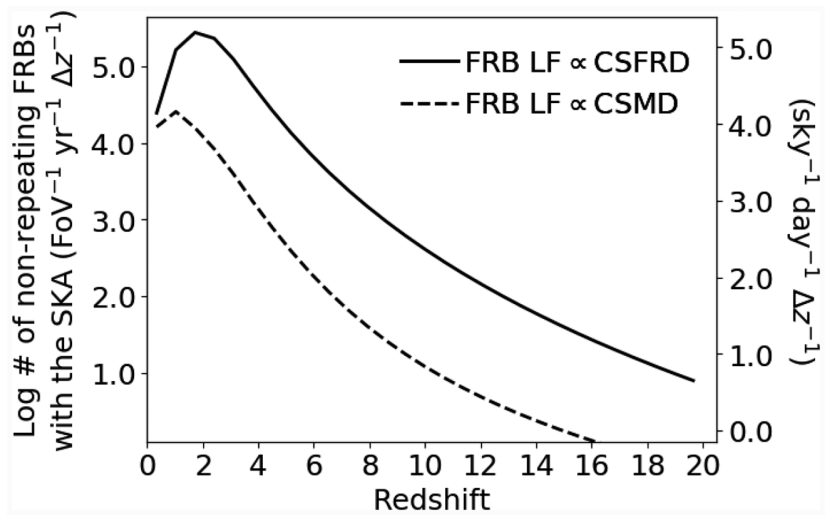

Figure 2. Expected number of non-repeating FRBs to be detected with the SKA phase 2 as a function of redshift. The number is in units of $\mathrm{FoV}^{-1}$ $\mathrm{yr}^{-1} \Delta z^{-1}$ (left-hand axis) and $\mathrm{sky}^{-1} \mathrm{~d}^{-1} \Delta z^{-1}$ (right-hand axis), where FoV is the field of view of the SKA Mid frequency aperture array. The solid and dashed lines correspond to different assumptions on redshift evolution of FRB LFs, that is, the CSFRD and CSMD, respectively. The characteristic spectral index of FRBs is assumed to be $\alpha=-0.3$. The observed frequency of SKA is $0.65 \mathrm{GHz}$. The pulse broadening of FRBs by scattering is empirically taken into consideration (see Hashimoto et al. 2020b, for details). No freefree absorption is assumed in the vicinity of FRB progenitors. The adopted Galactic coordinates of FRBs are $(\ell, b)=\left(45.0^{\circ},-90.0^{\circ}\right)$.

with $\Delta z=0.67$, mock FRBs are generated such that their number follows Fig. 2 for the CSMD and CSFRD cases. Within each redshift bin, the mock FRBs are distributed uniformly over the redshift. Their $\mathrm{DM}_{\mathrm{IGM}}$ are calculated by equation (3) assuming the three different cosmic reionization histories as shown in Fig. 1. Following the previous works in the literature (Kumar \& Linder 2019; Linder $2020)$, the line-of-sight fluctuation of $\mathrm{DM}_{\mathrm{IGM}}\left(\sigma_{\mathrm{DM}_{\mathrm{IGM}}}\right)$ is randomly added to $\mathrm{DM}_{\mathrm{IGM}}$ of the individual mock FRBs, assuming

$\sigma_{\mathrm{DM}_{\mathrm{IGM}}}=0.2 \frac{\mathrm{DM}_{\mathrm{IGM}}}{\sqrt{z}} \quad(z \leq 6)$.

At $z>6, \sigma_{\mathrm{DM}}^{2}(z>6)$ is expressed as $\sigma_{\mathrm{DM}_{\mathrm{IGM}}}^{2}(z=6)+\sigma_{\text {bubble }}^{2}(z)$ (Yoshiura \& Takahashi 2018), where $\sigma_{\text {bubble }}(z)$ is the variance of $\mathrm{DM}$ due to ionized bubbles in the IGM. The term $\sigma_{\text {bubble }}(z)$ is at most $\sim 170 \mathrm{pc} \mathrm{cm}^{-3}$ at $z>6$ (Yoshiura \& Takahashi 2018). This value contributes to $\sigma_{\mathrm{DM}_{\mathrm{IGM}}}(z>6)$ by only $\sim 10$ per cent under our assumption (equation 17). Therefore, we approximate $\sigma_{\mathrm{DM}_{\mathrm{IGM}}}(z>6)$ as $\sigma_{\mathrm{DM}_{\mathrm{IGM}}}(z=6)$ in this work.

The randomly added fluctuations of $\mathrm{DM}_{\mathrm{IGM}}$ follow a Gaussian probability distribution with the standard deviation of $\sigma_{\mathrm{DM}_{\mathrm{IGM}}}$ at each redshift bin. We note that $\mathrm{DM}_{\mathrm{obs}}$ is composed of contributions from the (i) interstellar medium in the Milky Way $\left(\mathrm{DM}_{\mathrm{MW}}\right)$, (ii) dark matter halo hosting the Milky Way $\left(\mathrm{DM}_{\text {halo }}\right)$, (iii) intervening galaxies $\left(\mathrm{DM}_{\text {inter, gal }}\right)$, (iv) IGM $\left(\mathrm{DM}_{\mathrm{IGM}}\right)$, and (v) galaxy hosting the FRB $\left(\mathrm{DM}_{\text {host }}\right)$. The $\mathrm{DM}_{\text {inter, gal }}$ has only a minor effect on $\mathrm{DM}_{\text {obs }}\left(\mathrm{DM}_{\text {inter, gal }}\right.$ $\lesssim 0.1 \mathrm{pc} \mathrm{cm}^{-3}$ on average at all $z<7$, Prochaska \& Neeleman 2018). Therefore, the $\mathrm{DM}_{\mathrm{IGM}}$ is measured by subtracting $\mathrm{DM}_{\mathrm{MW}}, \mathrm{DM}_{\text {halo }}$, and $\mathrm{DM}_{\text {host }}$ from $\mathrm{DM}_{\mathrm{obs}}$ (e.g. Hashimoto et al. 2020c). While the $\mathrm{DM}_{\text {halo }}$ and $\mathrm{DM}_{\text {host }}$ are poorly understood, their uncertainties (order of $10 \mathrm{pc} \mathrm{cm}^{-3}$ ) are much smaller than the $\mathrm{DM}_{\mathrm{IGM}}$ component (order of $10^{3} \mathrm{pc} \mathrm{cm}^{-3}$ ) and its line-of-sight fluctuation (order of $10^{2} \mathrm{pc} \mathrm{cm}^{-3}$ ) at the reionization epoch (e.g. Prochaska \& Zheng 2019). Therefore, we neglect the uncertainties of $\mathrm{DM}_{\text {halo }}$ and $\mathrm{DM}_{\text {host }}$ subtractions from the $\mathrm{DM}_{\mathrm{obs}}$ to derive the $\mathrm{DM}_{\mathrm{IGM}}$ observationally.

In terms of the $\mathrm{DM}_{\mathrm{obs}}$ uncertainty, the difference between currently detected low- $z$ FRBs $(z \lesssim 2)$ and future high- $z$ ones would be their slopes in the frequency-time space. The uncertainty of the slope corresponds to the $\mathrm{DM}_{\mathrm{obs}}$ uncertainty. For instance, Parkes detected FRBs up to $z \sim 2$ (e.g. Hashimoto et al. 2020c) with a spectral resolution of $\sim 400 \mathrm{kHz}$ (e.g. Keane \& Petroff 2015). The slope becomes $\sim 3$ times flatter from $z \sim 2$ to $\sim 20$. However, the SKA's spectral resolution is $\sim 10 \mathrm{kHz}$ (Torchinsky et al. 2016) which is $\gtrsim 10$ times better than the $400 \mathrm{kHz}$ spectral resolution of Parkes. Therefore, the SKA is expected to sample the slopes of high- $z$ FRBs as accurately as the current FRB observations or even better. A typical observational uncertainty of $\mathrm{DM}_{\mathrm{obs}}$ is $\lesssim 1$ per cent using current radio telescopes (e.g. Petroff et al. 2016). Therefore, we can reasonably ignore the $\mathrm{DM}_{\mathrm{obs}}$ uncertainty of mock FRBs in the SKA era.

We consider three cases of redshift measurements of FRBs: (A) spectroscopic redshift available for 100 per cent of the FRB sample, (B) spectroscopic redshift available for 10 per cent of the FRB sample, and (C) redshift estimated from an empirical relation between the time-integrated luminosity and duration of nonrepeating FRBs. Each case assumes two different redshift evolutions of FRB LFs (CSMD and CSFRD) and three different reionization histories as mentioned above.

\subsubsection{Mock data A: spectroscopic redshift for 100 per cent sample}

The spectroscopic redshifts of FRBs can be measured by identifying their host galaxies (e.g. Macquart et al. 2020) at FRBs' positions localized by the SKA (Torchinsky et al. 2016). We assume oneyear FRB detection with the SKA in this work. In contrast, the spectroscopic follow-up observations of FRB host galaxies can be performed over decades using future telescopes such as the European Extremely Large Telescope (Gilmozzi \& Spyromilio 2007) and the James Webb Space Telescope (Gardner et al. 2006). The SKA may also be able to measure spectroscopic redshifts of host galaxies at $z$ $\sim 10$ by detecting the $21-\mathrm{cm}$ emission line (e.g. Ghara, Choudhury \& Datta 2016). Therefore, future extensive efforts to measure spectroscopic redshifts of the host galaxies may significantly increase the number of FRBs with spectroscopic redshift. The number of such FRBs may approach the number of one-year FRB detection with the SKA. This case corresponds to the 100 per cent availability of the spectroscopic redshifts for one-year of FRB detection with the SKA. This is an optimistic assumption. However, it is useful for demonstrating the potential of FRBs in investigating the cosmic reionization history. Therefore, we show this case as the most ideal case. The redshift uncertainties of mock FRBs are approximated as $\sigma_{z}=0$ in this case. The generated mock FRB data (hereafter 'Mock data $A^{\prime}$ ) are shown in Fig. 3.

\subsubsection{Mock data B: spectroscopic redshift for 10 per cent sample}

FRB host identifications and their spectroscopic follow-up observations are expensive and time-consuming in general. Therefore, the spectroscopic redshifts will likely be available only for a part of the FRB sample. Currently, $\sim 10$ per cent of FRBs have their host identifications and spectroscopic redshifts ${ }^{1}$ (Heintz et al. 2020). For this mock data, we assume that the spectroscopic redshifts are available for 10 per cent of the FRBs detected with the SKA in one year. An analogy is the host identification for LGRBs. So far $\sim$ 2000 LGRBs have been localized down to arcminute level. ${ }^{2}$ Among them, $\sim 10$ per cent have their host identifications and spectroscopic

\footnotetext{
${ }^{1}$ http://frbhosts.org/

${ }^{2}$ https://www.mpe.mpg.de/ jeg/grbgen.html
} 


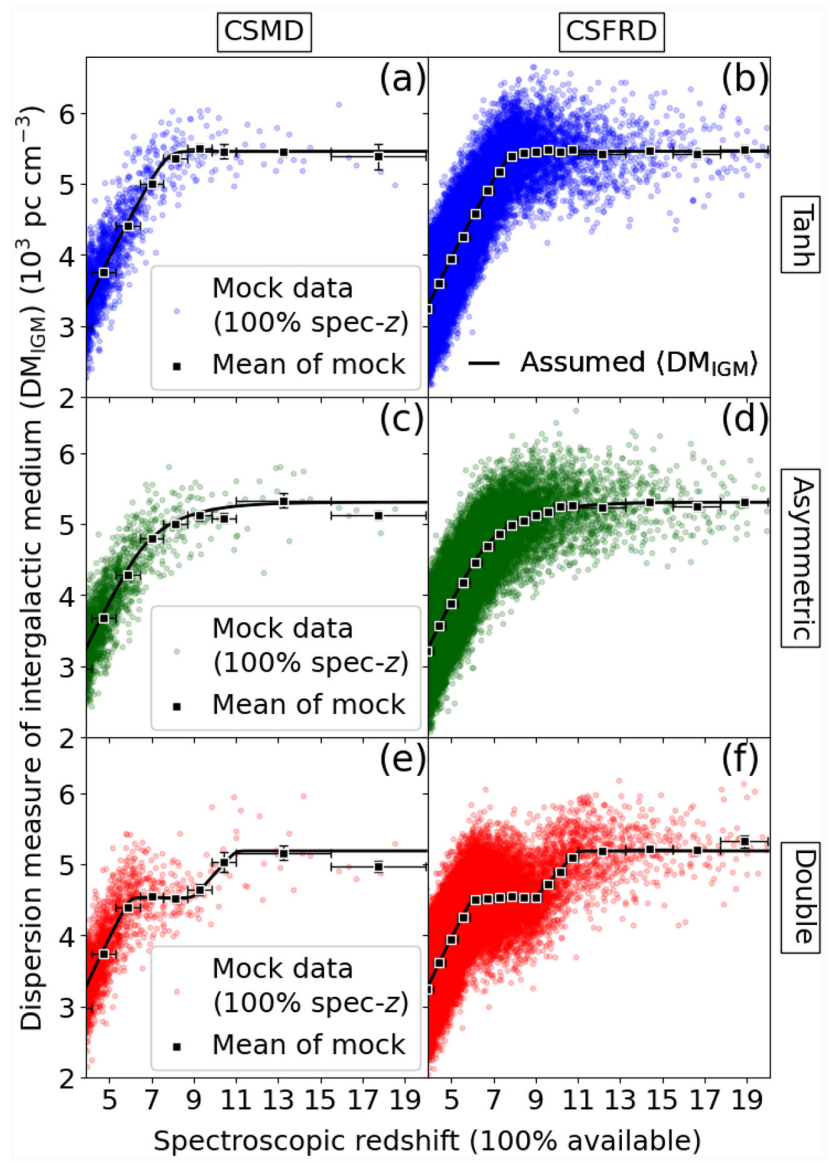

Figure 3. Simulated mock FRB data to be detected with the SKA (Mock data A). We assumed that spectroscopic redshifts are available for all of the mock data. The redshift uncertainty is ignored. From left- to right-hand panels, redshift evolutions of FRB LFs are assumed to be proportional to the CSMD and CSFRD, respectively. From top to bottom panels, Tanh, asymmetric, and double reionization histories are assumed, respectively. The black squares indicate averaged DMs of mock FRB data at redshift bins, $\left\langle\mathrm{DM}_{\mathrm{IGM} \text {, mock }}\right\rangle(z)$. The redshift bins are shown by horizontal bars. The vertical error bars indicate uncertainties of $\left\langle\mathrm{DM}_{\mathrm{IGM}}\right.$, mock $\rangle(z)$. These uncertainties are calculated by standard errors of mock data in the redshift bins, that is, $\sigma_{\mathrm{DM}}$ IGM mock $/ \sqrt{N_{\text {mock }}}$, where $\sigma_{\mathrm{DM}_{\mathrm{IGM} \text { mock }}}$ and $\sqrt{N_{\text {mock }}}$ are the standard deviation of $\mathrm{DM}_{\mathrm{IGM} \text {, mock }}$ and number of mock FRB data in each redshift bin.

redshifts ${ }^{3}$. The SKA will achieve better localizations for FRBs down to arcsecond level (Torchinsky et al. 2016). It is conservative to assume spectroscopic redshifts for 10 per cent of the future FRBs. In this case, mock data include 10 per cent of the originally generated FRBs with accurate redshift measurements, that is, $\sigma_{z}=0$. The generated mock FRB data (hereafter 'Mock data B') are shown in Fig. 4.

\subsubsection{Mock data C: redshift estimated from an empirical relation}

Hashimoto et al. (2019) reported an empirical relationship between the time-integrated luminosity $\left(L_{v}\right)$ and rest-frame intrinsic duration ( $w_{\text {int, rest }}$ ) of non-repeating FRBs (hereafter, $L_{v}-w_{\text {int, rest }}$ relation). The $L_{v}-w_{\text {int, rest }}$ relation can be used to constrain redshifts of individual FRBs without the host identification. The observed dispersion around

\footnotetext{
${ }^{3}$ http://www.grbhosts.org/
}

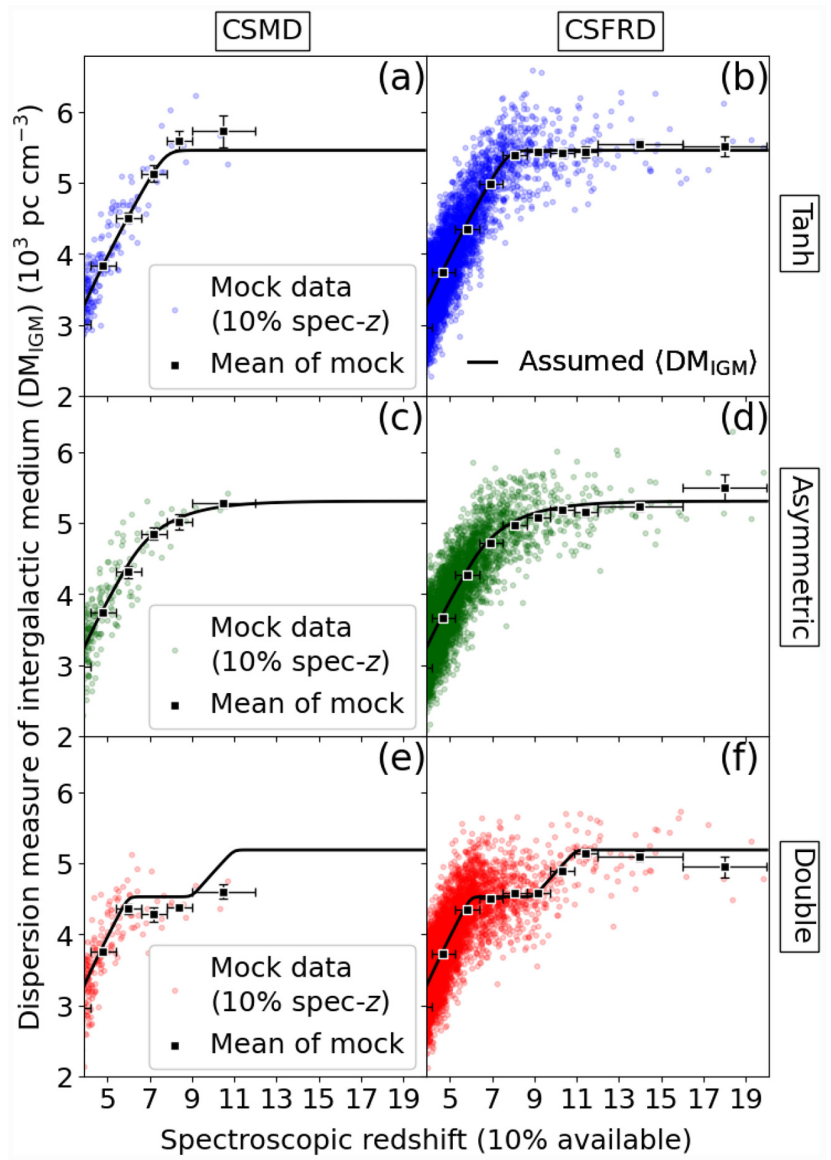

Figure 4. Same as Fig. 3 except that the spectroscopic redshift is available for 10 per cent of the mock FRB data (Mock data B).

the relation is $0.28 \mathrm{dex}$ in $\log \left(w_{\text {int, rest }}\right)$ axis. This dispersion is dominated by the observational uncertainties of the currently detected FRBs (Hashimoto et al. 2019). Since the observational uncertainties of SKA will be $\sim 2$ orders of magnitude smaller than that of currently working radio telescopes such as Parkes, the dispersion around the $L_{v}-w_{\text {int, rest }}$ relation would be significantly reduced from the current measurement of 0.28 dex. However, the dispersion may still be dominated by the intrinsic variation of FRBs rather than the observational uncertainty. In this work, we assume 10 per cent of the dispersion estimated for the current FRB sample. The 10 per cent dispersion of $0.28 \mathrm{dex}$ in $\log \left(w_{\text {int, rest }}\right)$ corresponds to a 0.13 dex dispersion in the log time-integrated luminosity (Hashimoto et al. 2019). This 0.13 dex dispersion is twice as large as the luminosity dispersion of type Ia supernovae since the dispersion of intrinsic luminosity of type Ia supernovae is $\sim 0.15 \mathrm{mag}$, that is, $0.15 / 2.5=0.06 \mathrm{dex}$ in $\log$ luminosity scale (e.g. Mohlabeng \& Ralston 2014; Pan et al. 2014).

The assumed $L_{v}-w_{\text {int, rest }}$ relation and its dispersion are shown in Fig. 5. The dotted lines in Fig. 5 indicate redshift tracks of FRBs with fixed observed fluences and durations. The redshift tracks are calculated from equation (6) in Hashimoto et al. (2019) and pulse broadening effect by redshift. Therefore, the intersection between the $L_{v}-w_{\text {int, rest }}$ relation and redshift track provides each FRB with a redshift estimate. The instrumental pulse broadening by the SKA is $\sim 1 \mathrm{~ms}$ for an FRB with $\mathrm{DM}_{\mathrm{obs}}=5 \times 10^{3} \mathrm{pc} \mathrm{cm}^{-1}$ (Hashimoto et al. 2020b). This value is negligible compared to the redshift broadening effect at the hydrogen reionization epoch (e.g. $11 \mathrm{~ms}$ for an FRB with $w_{\text {int, rest }}=1.0 \mathrm{~ms}$ at $z=10$ ). The redshift uncertainty 


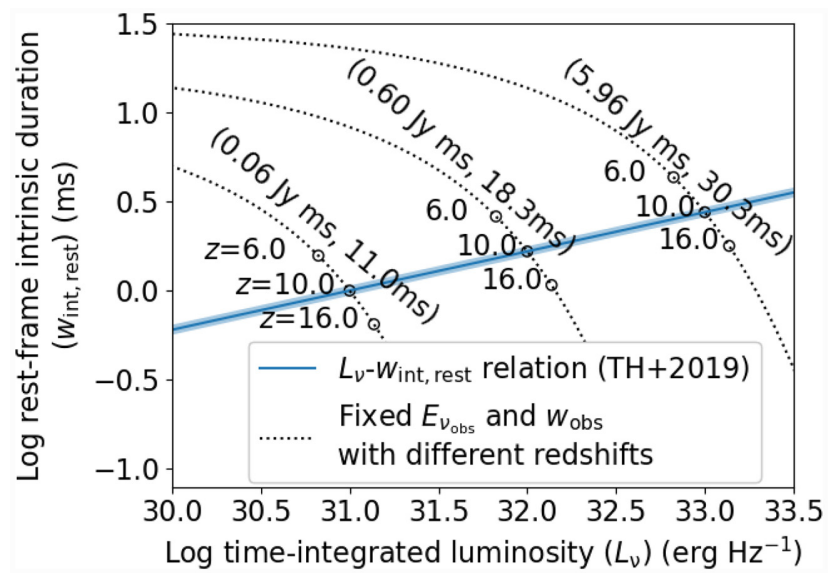

Figure 5. An empirical relation between the time-integrated luminosity and rest-frame intrinsic duration of FRBs $\left(L_{v}-w_{\text {int, rest }}\right.$ relation). The assumed dispersion of $L_{v}-w_{\text {int, rest }}$ relation in the SKA era is indicated by a shaded blue region, which is 10 per cent of the observed dispersion estimated from currently detected FRBs (Hashimoto et al. 2019). The dotted lines indicate redshift tracks of FRBs with fixed observed fluences $\left(E_{v_{\mathrm{obs}}}\right)$ and durations $\left(w_{\mathrm{obs}}\right)$ calculated from equation (6) in Hashimoto et al. (2019) and pulse broadening effect by redshift. The open circles labelled with redshift values correspond to $z=6.0,10.0$, and 16.0 on the tracks. The intersection between the $L_{v}-w_{\text {int, rest }}$ relation and dotted track provide each FRB with a redshift estimate. The presented $E_{v_{\mathrm{obs}}}$ and $w_{\mathrm{obs}}$ are examples of FRBs at $z=10$. The instrumental pulse broadening by the SKA is ignored since it is much smaller than the redshift broadening effect at the hydrogen reionization epoch.

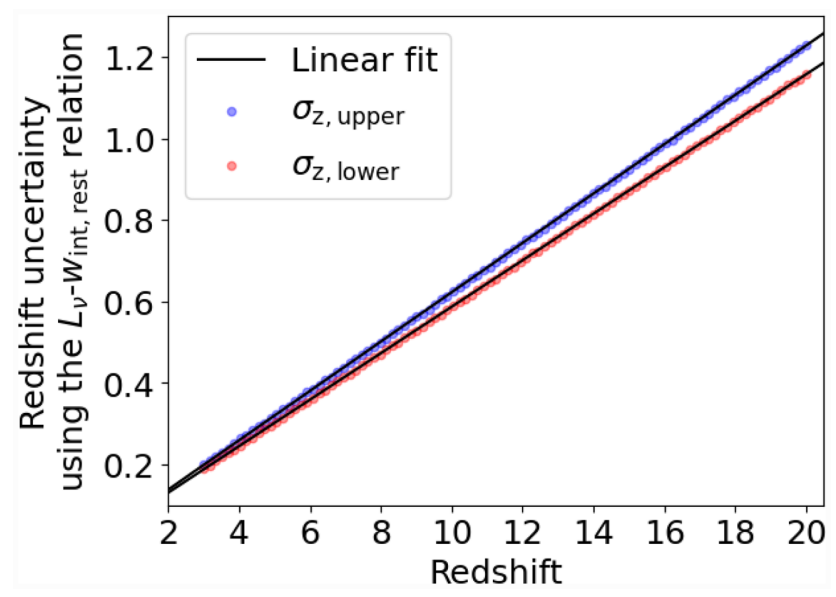

Figure 6. The redshift uncertainty of FRBs as a function of redshift using the empirical relation between the time-integrated luminosity and rest-frame intrinsic duration $\left(L_{v}-w_{\text {int, rest }}\right.$ relation). The blue and red dots represent upper and lower bounds of redshift uncertainties, respectively. These data points are calculated from overlapping regions between the dispersion of $L_{v}-w_{\text {int, rest }}$ relation and redshift tracks in Fig. 5. The solid lines indicate linear fits to the upper and lower bounds as a function of redshift.

is calculated as a function of redshift using the overlapping region between the dispersion of $L_{v}-w_{\text {int, rest }}$ relation (blue shaded region) and redshift tracks (dotted lines). The upper and lower bounds of redshift uncertainties ( $\sigma_{z \text {, upper }}$ and $\sigma_{\mathrm{z} \text {, lower }}$, respectively) are shown in Fig. 6 . The best-fitting linear functions to the uncertainties are

$\sigma_{\mathrm{z}, \mathrm{upper}}=0.061 z+0.017$

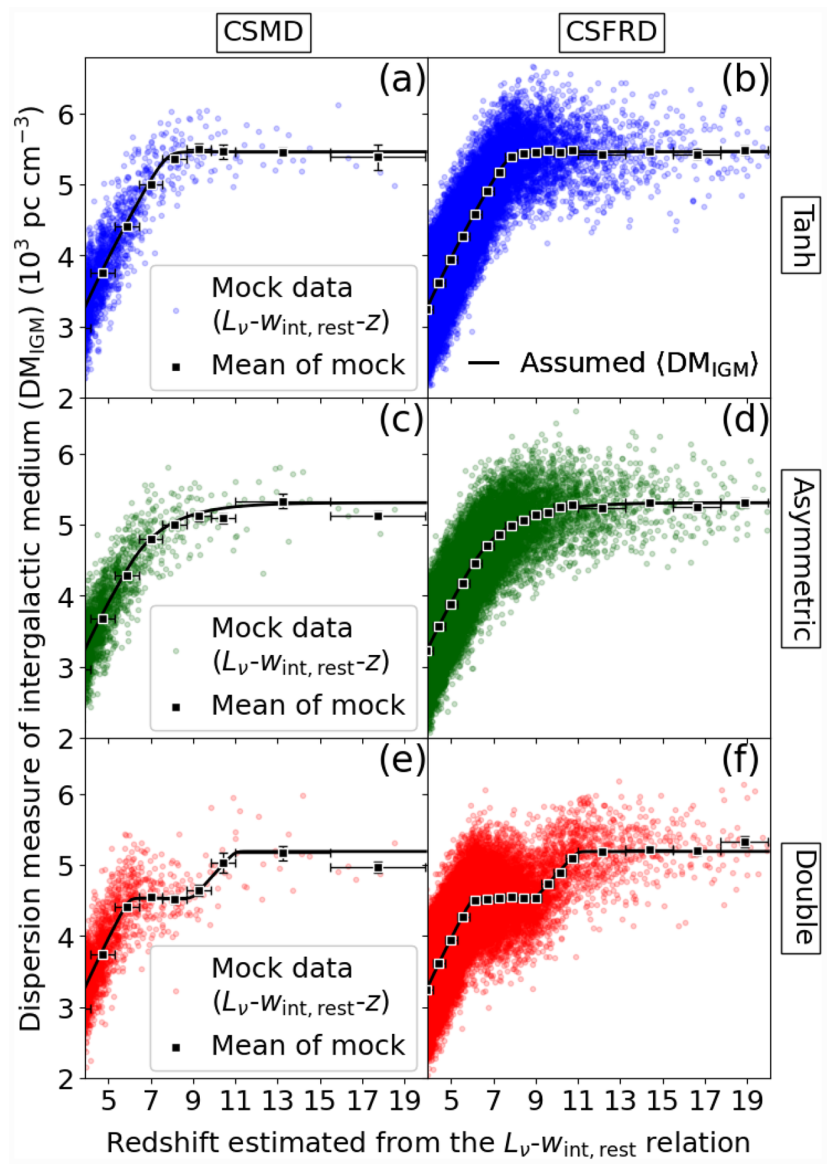

Figure 7. Same as Fig. 3 except that redshift uncertainties are randomly added to individual data using equations (18) and (19) (Mock data C).

and

$\sigma_{\mathrm{z}, \text { lower }}=0.057 z+0.017$.

Using equations (18) and (19), we randomly added redshift uncertainties to the mock FRB data. At each redshift, the randomized redshift errors follow an asymmetric Gaussian probability distribution which has the standard deviation of $\sigma_{\mathrm{z} \text {, upper }}$ for one side and $\sigma_{\mathrm{z} \text {, lower }}$ for the other side. The generated mock FRB data (hereafter 'Mock data C') are shown in Fig. 7.

\section{RESULTS}

Here, we reconstruct $X_{\mathrm{eH} I I}(z)$ from the mock FRB data described in Section 3. For this purpose, we calculated averaged $D_{\mathrm{IGM}}$ of mock FRB data, $\left\langle\mathrm{DM}_{\mathrm{IGM}}\right.$, mock $\rangle(z)$, at every redshift bin. The redshift bins are flexibly determined depending on the number of mock data. Mock data A has 7 (14) and 2 (4) bins at $3<z \leq$ 11 and at $11<z \leq 20$, respectively, for the CSMD (CSFRD) case. Mock data B has 5 (8) and 1 (2) bins at $3<z \leq 9$ (3 $<z \leq 12)$ and at $9<z \leq 12(12<z \leq 20)$, respectively, for the CSMD (CSFRD) case. The redshift bins of Mock data C are the same as that of Mock data A. $\left\langle\mathrm{DM}_{\mathrm{IGM} \text {, mock }}\right\rangle(z)$ are shown by black squares in Figs 3, 4, and 7. Their uncertainties are calculated by standard errors of mock FRB data in the redshift bins, that is, $\sigma_{\mathrm{DM}_{\mathrm{IGM}, \text { mock }}} / \sqrt{N_{\text {mock }}}$, where $\sigma_{\mathrm{DM}_{\mathrm{IGM}, \text { mock }}}$ and $\sqrt{N_{\text {mock }}}$ are the standard deviation of $\mathrm{DM}_{\mathrm{IGM}}$, mock and the number of mock FRB data in each redshift bin. The measured $\left\langle\mathrm{DM}_{\mathrm{IGM} \text {, mock }}\right\rangle(z)$ was used to estimate 


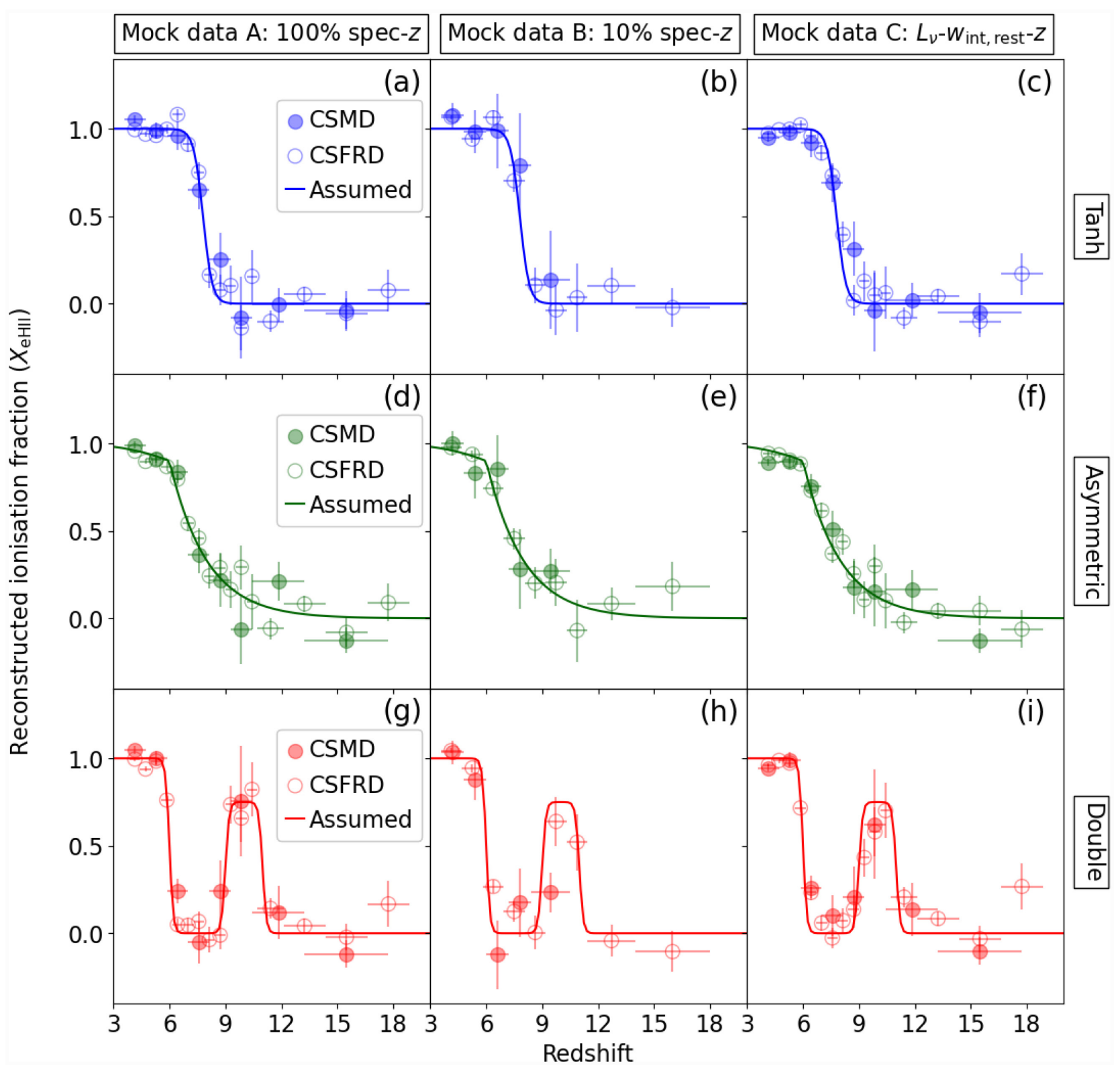

Figure 8. Ionization fractions as a function of redshift, $X_{\mathrm{eH} \mathrm{II}}(z)$, reconstructed from mock FRB data. The reconstructed data are shown by filled and open circles. The filled and open circles correspond to assumptions on redshift evolutions of FRB LFs which are proportional to the CSMD and CSFRD, respectively. The horizontal bars indicate redshift bins. The vertical bars indicate uncertainties of $X_{\mathrm{eH}}$ II which include uncertainties of $\left\langle\mathrm{DM}_{\mathrm{IGM}}\right.$, mock $\rangle(z)$ in Figs 3,4 , and 7. The prior assumptions on reionization histories are shown by solid lines. From left- to right-hand panels, Mock data A (spectroscopic redshift available for 100 per cent of FRB sample), B (spectroscopic redshift available for 10 per cent of FRB sample), and C (redshift estimated from an empirical relation between the time-integrated luminosity and rest-frame intrinsic duration of non-repeating FRBs: $L_{v}-w_{\text {int, }}$ rest relation) are shown, respectively. From top to bottom panels, Tanh, asymmetric, and double reionization histories are used as prior assumptions, respectively.

the slopes of the mock FRB data, $\frac{\mathrm{d}\left(\mathrm{DM}_{\mathrm{IGM}, \text { mock }}\right)(z)}{\mathrm{d} z}$, in Figs 3,4 , and 7. This slope measurement allows us to reconstruct $X_{\mathrm{eH} \mathrm{II}}(z)$ using equation (5). The reconstructed $X_{\mathrm{eH} \mathrm{II}}(z)$ are shown in Fig. 8. The three reionization histories and three cases of redshift measurements (Mock data A, B, and C) correspond to nine panels in Fig. 8. The dominant source of the errors in Fig. 8 is the uncertainty of $\left\langle\mathrm{DM}_{\mathrm{IGM}}\right\rangle$ in each redshift bin. This uncertainty is calculated by a standard error in each redshift bin in Figs 3, 4, and 7 as mentioned above. In all of the cases, the assumed reionization histories are reasonably reproduced. We did not assume any functional shape of the reionization history when $X_{\mathrm{eH} \text { II }}$ was reconstructed from the mock FRB data. Even the shape of double reionization can be reconstructed (bottom panels in Fig. 8), which indicates the capability of future FRBs and the feasibility of our method.

\section{DISCUSSION}

\subsection{Reionization history reconstructed from mock FRB data}

The CMB observations constrain $\tau_{\mathrm{CMB}}$ (e.g. Pagano et al. 2020). However, the ionization fraction as a function of redshift is difficult to be measured solely by using $\tau_{\mathrm{CMB}}$ since $\tau_{\mathrm{CMB}}$ is an integrated value over redshift $z=0$ to $\sim 1100$. In this sense, solely using $\tau_{\mathrm{CMB}}$ is not 
sensitive to distinguishing between the three reionization histories with the same $\tau_{\mathrm{CMB}}$ value presented in this work. We note that the detailed redshift evolution of the ionization fraction is imprinted in the shape of the power spectrum of CMB polarization (Zaldarriaga et al. 1997; Kaplinghat et al. 2003; Planck Collaboration VI 2020). Therefore, the CMB polarization can constrain the high- $z$ end of reionization (e.g. Planck Collaboration VI 2020).

The $\mathrm{DM}_{\mathrm{IGM}}$ of FRBs is a similar parameter to $\tau_{\mathrm{CMB}}$. In previous studies (e.g. Ioka 2003; Inoue 2004; Fialkov \& Loeb 2016), the $\mathrm{DM}_{\mathrm{IGM}}$ has been proposed as a tool to constrain the reionization history independently from the $\tau_{\mathrm{CMB}}$ measurement. Fialkov \& Loeb (2016) showed that different reionization histories give rise to different shapes of $\mathrm{DM}_{\mathrm{IGM}}$ as a function of redshift. Therefore, having a large sample of FRBs would improve the constraint on the redshift evolution of the cosmic ionized fraction. However, $\mathrm{d}\left\langle\mathrm{DM}_{\mathrm{IGM}}\right\rangle / \mathrm{d} z$ has been rarely discussed in spite of its better sensitivity to the reionization history than $\operatorname{DM}_{\mathrm{IGM}}(z)$ itself (Zheng et al. 2014). In this work, we emphasize that the advantage of FRBs over CMB is the availability of $\mathrm{DM}_{\mathrm{IGM}}$ as a function of redshift, that is, availability of $\mathrm{d}\left\langle\mathrm{DM}_{\mathrm{IGM}}\right\rangle / \mathrm{d} z$. By taking advantage of FRBs, we will be able to directly measure the ionization history as a function of redshift. Fig. 8 indicates that future FRBs are able to distinguish between these histories using our method. Having only 10 per cent of the spectroscopic redshift measurements of the SKA FRBs might be enough to reveal the cosmic reionization history.

Recently, Dai \& Xia (2020) proposed to use an autocorrelation power spectrum of $\mathrm{DM}_{\mathrm{IGM}}$ measurements to constrain the reionization epoch. Based on their mock FRB data, they predicted that the duration of the transition phase of reionization will be significantly constrained compared to using the CMB data only. The advantage of this method is that accurate redshift measurements are not necessary but a rough redshift distribution is enough for the analysis. However, their method has to assume a functional shape of the reionization history which is poorly known. On the other hand, our method requires the redshift measurements for individual FRBs, but it is able to measure the cosmic reionization history as it is without the shape assumption. Motivated by Dai \& Xia (2020), redshift information of FRBs may be obtained by a spatial cross-correlation between FRBs and high- $z$ galaxies whose redshifts are known from galaxy surveys (e.g. Hsiao et al., in preparation). This may provide FRBs at each $\mathrm{DM}_{\mathrm{IGM}}$ bin with a representative redshift, which will work in a similar way to our method.

\subsection{Assumptions in the luminosity-duration relation}

The empirically derived $L_{v}-w_{\text {int, rest }}$ relation of non-repeating FRBs (Hashimoto et al. 2019) is used to estimate the redshifts of Mock data C. We caution that there are two assumptions behind Mock data C.

One is the reduction of dispersion around the relation in the SKA era. As we mentioned in Section 3.3.3, the dispersion of the relation is dominated by the observational uncertainties for the current FRB sample (Hashimoto et al. 2019). The observational uncertainties will decrease significantly for the FRBs detected with the SKA phase 2 (e.g. Torchinsky et al. 2016). The dispersion around the relation would be dominated by the intrinsic variation of FRBs in the SKA era. Indeed some FRB models predict positive correlations between the time-integrated luminosity and duration (e.g. Lyubarsky 2014; Geng \& Huang 2015; Romero, del Valle \& Vieyro 2016). In these models, the time-integrated luminosity depends not only on the duration but also on other physical parameters. Therefore, one possible way to reduce the intrinsic dispersion is by introducing (an) additional factor(s) to the relation. In the past, the peak luminosity of type Ia supernovae has been reported with a dispersion of $0.8 \mathrm{mag}$ in the $B$ band (Phillips 1993). So far, extensive efforts were made to reduce the dispersion by taking into account new factors such as light-curve shape, colour, and host properties of type Ia supernovae (e.g. Pan et al. 2014). Such corrections for the relation allowed us to utilize type Ia supernovae as a standard candle eventually. Similarly, substantial efforts will be necessary for the redshift estimate using the $L_{v}-w_{\text {int, rest }}$ relation.

Another assumption is the 'no redshift evolution' of the $L_{v}-w_{\text {int, rest }}$ relation. If the relation depends on redshift, the redshift measured from the $L_{v}-w_{\text {int, rest }}$ relation contains a systematic uncertainty. Unless the relation is corrected for the evolution, the derived reionization history will systematically shift towards lower/higher redshifts while the shape of the ionization history may be less affected. For the redshift-measurement purpose, the $L_{v}-w_{\text {int, rest }}$ relation also needs to be well established as a function of redshift.

\section{CONCLUSION}

We propose a method to directly measure the ionization fraction of the IGM as a function of redshift using FRBs. The DM in the IGM $\left(\mathrm{DM}_{\mathrm{IGM}}\right)$ derived from FRBs is unique because it enables us to measure the integrated electron densities in the IGM at different redshifts. The differential of averaged $\mathrm{DM}_{\mathrm{IGM}}$ against redshift, $\mathrm{d}\left\langle\mathrm{DM}_{\mathrm{IGM}}\right\rangle / \mathrm{d} z$, is proportional to the ionization fraction. Therefore, $\mathrm{d}\left\langle\mathrm{DM}_{\mathrm{IGM}}\right\rangle / \mathrm{d} z$ allows us to directly measure the ionization fraction of the IGM as a function of redshift. We consider future non-repeating FRB sources to be detected with the SKA at the cosmic reionization epoch. For a demonstration purpose, three cosmic ionization histories including Tanh, asymmetric, and double reionization are assumed as priors. Since these histories are parametrized with the same Thomsonscattering optical depth $\left(\tau_{\mathrm{CMB}}\right)$, solely using $\tau_{\mathrm{CMB}}$ constrained from the CMB data is not sensitive to distinguishing between these histories. Based on mock FRB data in the SKA era, we found that the cosmic reionization history can be reasonably reconstructed by using $\mathrm{d}\left\langle\mathrm{DM}_{\mathrm{IGM}}\right\rangle / \mathrm{d} z$ for each reionization case. In the reconstruction process, no assumption is made on a functional shape of the cosmic reionization history. The advantage of our method is that it allows us to measure the reionization history as it is, in contrast to previously proposed methods solely using $\tau_{\mathrm{CMB}}$ or $\mathrm{DM}_{\mathrm{IGM}}$. Our results indicate the capability of future FRBs and the feasibility of our method in revealing the cosmic reionization history.

\section{ACKNOWLEDGEMENTS}

We are very grateful to the anonymous referee for many insightful comments. We thank Susumu Inoue and Shintaro Yoshiura for useful discussions. TH and AYLO are supported by the Centre for Informatics and Computation in Astronomy (CICA) at National Tsing Hua University (NTHU) through a grant from the Ministry of Education of Taiwan. TG acknowledges the support by the Ministry of Science and Technology of Taiwan through grant 108-2628M-007-004-MY3. AYLO's visit to NTHU was supported by the Ministry of Science and Technology of the ROC (Taiwan) grant 105-2119-M-007-028-MY3, hosted by Albert Kong. This work used high-performance computing facilities operated by the CICA at NTHU. This equipment was funded by the Ministry of Education of Taiwan, the Ministry of Science and Technology of Taiwan, and NTHU. This research has made use of NASA's Astrophysics Data System. 


\section{DATA AVAILABILITY}

The data underlying this article will be shared on reasonable request to the corresponding author.

\section{REFERENCES}

Bañados E. et al., 2018, Nature, 553, 473

Barkana R., 2016, Phys. Rep., 645, 1

Barkana R., Loeb A., 2001, Phys. Rep., 349, 125

Barnett R., Warren S. J., Becker G. D., Mortlock D. J., Hewett P. C., McMahon R. G., Simpson C., Venemans B. P., 2017, A\&A, 601, A16

Becker G. D., Bolton J. S., Haehnelt M. G., Sargent W. L. W., 2011, MNRAS, 410, 1096

Becker G. D., Bolton J. S., Madau P., Pettini M., Ryan-Weber E. V., Venemans B. P., 2015, MNRAS, 447, 3402

Bosman S. E. I., Fan X., Jiang L., Reed S., Matsuoka Y., Becker G., Haehnelt M., 2018, MNRAS, 479, 1055

Bouwens R. J. et al., 2012, ApJ, 752, L5

Bowman J. D., Rogers A. E. E., 2010, Nature, 468, 796

Bowman J. D., Rogers A. E. E., Hewitt J. N., 2008, ApJ, 676, 1

Bowman J. D., Rogers A. E. E., Monsalve R. A., Mozdzen T. J., Mahesh N., 2018, Nature, 555, 67

Bromm V., Yoshida N., 2011, ARA\&A, 49, 373

Caleb M., Flynn C., Stappers B. W., 2019, MNRAS, 485, 2281

Cen R., 2003, ApJ, 591, 12

Chawla P. et al., 2017, ApJ, 844, 140

Chevallier M., Polarski D., 2001, Int. J. Mod. Phys. D, 10, 213

Cordes J. M., Chatterjee S., 2019, ARA\&A, 57, 417

Dai J.-P., Xia J.-Q., 2020, preprint (arXiv:2004.11276)

Douspis M., Aghanim N., Ilić S., Langer M., 2015, A\&A, 580, L4

Duncan K., Conselice C. J., 2015, MNRAS, 451, 2030

Fan X. et al., 2006, AJ, 132, 117

Fan X., Narayanan V. K., Strauss M. A., White R. L., Becker R. H., Pentericci L., Rix H.-W., 2002, AJ, 123, 1247

Fialkov A., Loeb A., 2016, J. Cosmol. Astropart. Phys., 2016, 004

Fialkov A., Loeb A., 2017, ApJ, 846, L27

Finkelstein S. L. et al., 2012, ApJ, 758, 93

Furlanetto S. R., Oh S. P., Briggs F. H., 2006, Phys. Rep., 433, 181

Gardner J. P. et al., 2006, Space Sci. Rev., 123, 485

Geng J. J., Huang Y. F., 2015, ApJ, 809, 24

Ghara R., Choudhury T. R., Datta K. K., 2016, MNRAS, 460, 827

Gilmozzi R., Spyromilio J., 2007, The Messenger, 127, 11

Goto T., 2006, MNRAS, 371, 769

Hartoog O. E. et al., 2015, A\&A, 580, A139

Hashimoto T. et al., 2020b, MNRAS, 497, 4107

Hashimoto T. et al., 2020c, MNRAS, 498, 3927

Hashimoto T., Goto T., Wang T.-W., Kim S. J., Wu Y.-H., Ho C.-C., 2019, MNRAS, 488, 1908

Hashimoto T., Goto T., Wang T.-W., Kim S. J., Ho S. C. C., On A. Y. L., Lu T.-Y., Santos D. J. D., 2020a, MNRAS, 494, 2886

Hassan S., Davé R., Mitra S., Finlator K., Ciardi B., Santos M. G., 2018, MNRAS, 473, 227

Heintz K. E. et al., 2020, ApJ, 903, 152

Hinshaw G. et al., 2013, ApJS, 208, 19

Inoue S., 2004, MNRAS, 348, 999

Ioka K., 2003, ApJ, 598, L79

Kaplinghat M., Chu M., Haiman Z., Holder G. P., Knox L., Skordis C., 2003, ApJ, 583, 24

Keane E. F., Petroff E., 2015, MNRAS, 447, 2852
Kit Lau A. W., Mitra A., Shafiee M., Smoot G., 2020, preprint (arXiv:2006.11072)

Kumar P., Linder E. V., 2019, Phys. Rev. D, 100, 083533

Lacey C. G., Baugh C. M., Frenk C. S., Benson A. J., 2011, MNRAS, 412, 1828

Lewis A., 2008, Phys. Rev. D, 78, 023002

Linder E. V., 2003, Phys. Rev. Lett., 90, 091301

Linder E. V., 2020, Phys. Rev. D, 101, 103019

López Fernández R. et al., 2018, A\&A, 615, A27

Lorimer D. R., Bailes M., McLaughlin M. A., Narkevic D. J., Crawford F., 2007, Science, 318, 777

Lu T.-Y. et al., 2020, ApJ, 893, 69

Lyubarsky Y., 2014, MNRAS, 442, L9

Macquart J. P. et al., 2020, Nature, 581, 391

Madau P., Fragos T., 2017, ApJ, 840, 39

Matsuoka Y. et al., 2018, ApJ, 869, 150

Meiksin A. A., 2009, Rev. Mod. Phys., 81, 1405

Melandri A. et al., 2015, A\&A, 581, A86

Mohlabeng G. M., Ralston J. P., 2014, MNRAS, 439, L16

Naidu R. P., Tacchella S., Mason C. A., Bose S., Oesch P. A., Conroy C., 2020, ApJ, 892, 109

Onoue M. et al., 2017, ApJ, 847, L15

Pagano L., Delouis J. M., Mottet S., Puget J. L., Vibert L., 2020, A\&A, 635, A99

Pan Y. C. et al., 2014, MNRAS, 438, 1391

Perley D. A. et al., 2016, ApJ, 817, 7

Petroff E. et al., 2016, PASA, 33, e045

Phillips M. M., 1993, ApJ, 413, L105

Planck Collaboration XIII, 2016, A\&A, 594, A13

Planck Collaboration VI, 2020, A\&A, 641, A6

Pritchard J. R., Loeb A., 2012, Rep. Prog. Phys., 75, 086901

Prochaska J. X., Neeleman M., 2018, MNRAS, 474, 318

Prochaska J. X., Zheng Y., 2019, MNRAS, 485, 648

Robertson B. E., Ellis R. S., Furlanetto S. R., Dunlop J. S., 2015, ApJ, 802, L19

Rogers A. E. E., Bowman J. D., 2008, AJ, 136, 641

Romero G. E., del Valle M. V., Vieyro F. L., 2016, Phys. Rev. D, 93, 023001

Salvador-Solé E., Manrique A., Guzman R., Rodríguez Espinosa J. M., Gallego J., Herrero A., Mas-Hesse J. M., Marín Franch A., 2017, ApJ, 834, 49

Shull J. M., Smith B. D., Danforth C. W., 2012, ApJ, 759, 23

Springel V. et al., 2005, Nature, 435, 629

Torchinsky S. A., Broderick J. W., Gunst A., Faulkner A. J., van Cappellen W., 2016, preprint (arXiv:1610.00683)

Totani T. et al., 2014, PASJ, 66, 63

Totani T., Kawai N., Kosugi G., Aoki K., Yamada T., Iye M., Ohta K., Hattori T., 2006, PASJ, 58, 485

Wang F. et al., 2019, ApJ, 884, 30

Yang J. et al., 2020, ApJ, 897, L14

Yoshiura S., Takahashi K., 2018, MNRAS, 473, 1570

Yoshiura S., Hasegawa K., Ichiki K., Tashiro H., Shimabukuro H., Takahashi K., 2017, MNRAS, 471, 3713

Zaldarriaga M., Spergel D. N., Seljak U., 1997, ApJ, 488, 1

Zheng Z., Ofek E. O., Kulkarni S. R., Neill J. D., Juric M., 2014, ApJ, 797, 71

Zhou B., Li X., Wang T., Fan Y.-Z., Wei D.-M., 2014, Phys. Rev. D, 89, 107303

This paper has been typeset from a $\mathrm{T}_{\mathrm{E}} \mathrm{X} / \mathrm{LAT}_{\mathrm{E}} \mathrm{X}$ file prepared by the author. 